\title{
ERRATUM
}

\section{Predictors of 30-day hospital readmission after mechanical thrombectomy for acute ischemic stroke}

TO THE READERSHIP: An error appeared in the article by Mouchtouris et al. (Mouchtouris N, Al Saiegh F, Valcarcel B, et al. Predictors of 30-day hospital readmission after mechanical thrombectomy for acute ischemic stroke. J Neurosurg. Published online May 1, 2020. doi:10.3171/2020.2.JNS193249).

Several statistical inaccuracies appeared in the article. In the Results section of the abstract, the following sentence was revised.

The median time to readmission was 10.5 days (IQR 6.0-14.3).

In the Patient Characteristics section of the Results in the text, the following sentence was revised.

The median hospital LOS was 7.0 days (IQR

$4.0-11.0)$ and the median length of ICU stay was

3.0 days (IQR 2.0-5.0).

In the Outcomes section of the Results, the following sentences were revised.

The median time to readmission was 10.5 days after discharge (IQR 6.0-14.3). The mean LOS during the readmission was 6.0 days (IQR 3.010.3).

The median index LOS was 7.0 days (IQR 5.8$14.5)$ in the patients who were later readmitted and 6.0 days (IQR 4.0-11.0) in those who were not readmitted $(\mathrm{p}=0.045)$.
Finally, several values in Tables 1 and 2 were updated. These values are shown in bold in the tables that appear on the following pages.

The article has been corrected online as of August 21, 2020 .

Nikolaos Mouchtouris, MD

M. Reid Gooch, MD

Thomas Jefferson University and Jefferson Hospital for Neuroscience,

Philadelphia, PA

CORRESPONDING ARTICLE See pp 1500-1504.

INCLUDE WHEN CITING

Published online August 21, 2020; DOI: 10.3171/2020.7.JNS193249a.

CAANS 2021, except where prohibited by US copyright law 
TABLE 1. Baseline patient characteristics

\begin{tabular}{|c|c|c|c|}
\hline Characteristic & $\begin{array}{l}\text { Readmitted } \\
\quad(n=42)\end{array}$ & $\begin{array}{l}\text { Not Readmitted } \\
\quad(n=519)\end{array}$ & $\begin{array}{c}p \\
\text { Value }\end{array}$ \\
\hline Sex & & & 0.118 \\
\hline Male & $33.3 \%$ & $45.9 \%$ & \\
\hline Female & $66.7 \%$ & $54.1 \%$ & \\
\hline Age, yrs & $74.3 \pm 12.5$ & $71.8 \pm 15.7$ & 0.308 \\
\hline \multicolumn{4}{|l|}{ LOS, days } \\
\hline Hospital, median (IQR) & $7.0(5.8-14.5)$ & $6.0(4.0-11.0)$ & 0.045 \\
\hline ICU, median (IQR) & $2.0(2.0-7.0)$ & $3.0(2.0-5.0)$ & 0.901 \\
\hline \multicolumn{4}{|l|}{ Comorbidities } \\
\hline HTN & $85.7 \%$ & $69.4 \%$ & 0.026 \\
\hline Diabetes mellitus & $21.4 \%$ & $21.5 \%$ & 0.996 \\
\hline COPD & $7.1 \%$ & $6.6 \%$ & 0.897 \\
\hline Coronary artery disease & $19.0 \%$ & $16.4 \%$ & 0.665 \\
\hline Peripheral vascular disease & $19.0 \%$ & $8.7 \%$ & 0.029 \\
\hline $\mathrm{CHF}$ & $19.0 \%$ & $12.3 \%$ & 0.215 \\
\hline Hyperlipidemia & $54.8 \%$ & $39.0 \%$ & 0.047 \\
\hline Stroke history & $21.4 \%$ & $13.0 \%$ & 0.130 \\
\hline NIHSS at presentation & $16.7 \pm 6.7$ & $15.0 \pm 6.2$ & 0.310 \\
\hline IV tPA & $45.2 \%$ & $46.6 \%$ & 0.868 \\
\hline $\mathrm{mTICl}$ score & & & 0.936 \\
\hline 0 & $4.8 \%$ & $3.7 \%$ & \\
\hline 1 & $0 \%$ & $1.6 \%$ & \\
\hline $2 A$ & $7.1 \%$ & $6.4 \%$ & \\
\hline $2 B$ & $14.3 \%$ & $16.7 \%$ & \\
\hline 3 & $73.8 \%$ & $71.7 \%$ & \\
\hline $\begin{array}{l}\text { Hemorrhagic conversion } \\
\text { (grades } 3 \text { \& 4) }\end{array}$ & $21.4 \%$ & $11.2 \%$ & 0.052 \\
\hline
\end{tabular}

$\mathrm{CHF}=$ congestive heart failure; $\mathrm{COPD}=$ chronic obstructive pulmonary disease; HTN = hypertension; IV = intravenous.

Values are presented as percentage of patients or mean \pm SD unless otherwise indicated.
TABLE 2. Patient readmission data

\begin{tabular}{|c|c|c|c|}
\hline $\begin{array}{l}\text { Reason for } \\
\text { Readmission }\end{array}$ & $\begin{array}{l}\text { No. of } \\
\text { Patients } \\
(\%)\end{array}$ & $\begin{array}{c}\text { Time to } \\
\text { Readmission, } \\
\text { Median (IQR) }\end{array}$ & $\begin{array}{l}\text { Readmission } \\
\text { LOS, Median } \\
\text { (IQR) }\end{array}$ \\
\hline $\begin{array}{l}\text { Infection (pneumonia, } \\
\text { UTI, sepsis) }\end{array}$ & $14(33.3 \%)$ & $8.0(4.5-12.3)$ & $6.5(3.0-9.8)$ \\
\hline Cerebrovascular event & $7(16.7 \%)$ & $13.0(10.0-17.0)$ & $6.0(6.0-19.0)$ \\
\hline New infarct & $6(14.3 \%)$ & & \\
\hline $\mathrm{ICH}$ & $1(2.4 \%)$ & & \\
\hline $\begin{array}{l}\text { Cardiovascular event } \\
\text { (Afib w/ RVR, HTN } \\
\text { urgency, CHF } \\
\text { exacerbation) }\end{array}$ & $8(19.1 \%)$ & $13.0(6.8-21.5)$ & $4.0(3.0-9.5)$ \\
\hline DVT or PE & $3(7.1 \%)$ & $7.0(1.0-7.0)$ & $10.0(6.0-10.0)$ \\
\hline $\begin{array}{l}\text { Other (seizures, GI } \\
\text { bleed, rehab limita- } \\
\text { tions) }\end{array}$ & $9(21.4 \%)$ & $12.0(1.0-13.5)$ & $6.0(3.0-16.0)$ \\
\hline Pseudoaneurysm & $1(2.4 \%)$ & 4 & 7 \\
\hline
\end{tabular}

Afib = atrial fibrillation; $\mathrm{GI}=$ gastrointestinal; $\mathrm{ICH}=$ intracerebral hemorrhage; rehab = rehabilitation; RVR = rapid ventricular response.

Time and LOS values presented in days. 